\title{
Social-Aware Multicast in Disruption-Tolerant Networks
}

\author{
Wei Gao, Student Member, IEEE, Qinghua Li, Student Member, IEEE, Bo Zhao, Student Member, IEEE, and \\ Guohong Cao, Fellow, IEEE
}

\begin{abstract}
Node mobility and end-to-end disconnections in disruption-tolerant networks (DTNs) greatly impair the effectiveness of data forwarding. Although social-based approaches can address the problem, most existing solutions only focus on forwarding data to a single destination. In this paper, we study multicast with single and multiple data items in DTNs from a social network perspective, develop analytical models for multicast relay selection, and furthermore investigate the essential difference between multicast and unicast in DTNs. The proposed approach selects relays according to their capabilities, measured by social-based metrics, for forwarding data to the destinations. The design of social-based metrics exploits social network concepts such as node centrality and social community, and the selected relays ensure achieving the required data delivery ratio within the given time constraint. Extensive trace-driven simulations show that the proposed approach has similar data delivery ratio and delay to that of Epidemic routing, but significantly reduces data forwarding cost, measured by the number of relays used.
\end{abstract}

Index Terms-Centrality, community, disruption-tolerant network (DTN), multicast, social network.

\section{INTRODUCTION}

I N DISRUPTION-TOLERANT networks (DTNs) [13], mobile users contact each other opportunistically. Due to low node density and unpredictable node mobility, end-to-end connections are difficult to maintain in such networks. Alternatively, exploitation of node mobility allows nodes to physically carry data as relays and forward data opportunistically upon contact. The key problem for effective data forwarding is, therefore, selection of the appropriate nodes as relays.

Social network analysis (SNA) has been exploited for data forwarding in DTNs [10], [20], [30], [36]. There are two key concepts in SNA: 1) Community, which is naturally formed according to social relations among people; social community is the natural outcome from the "small-world" phenomenon, which is formalized as a random graph problem in [39]. 2) Centrality, which shows that some nodes in a community are the common acquaintances of other nodes and act as communication hubs. Since social relations among mobile users are

Manuscript received December 29, 2010; revised July 20, 2011; October 15, 2011; and December 03, 2011; accepted December 21, 2011; approved by IEEE/ACM TRANSACTIONS ON NETWORKING Editor D. Starobinski. Date of publication January 31, 2012; date of current version October 11, 2012. This work was supported in part by Network Science CTA under Grant W911NF-09-2-0053.

The authors are with the Department of Computer Science and Engineering, The Pennsylvania State University, University Park, PA 16802 USA (e-mail: weigao@cse.psu.edu; qx1118@cse.psu.edu; bzhao@cse.psu.edu; gcao@cse.psu.edu).

Color versions of one or more of the figures in this paper are available online at http://ieeexplore.ieee.org.

Digital Object Identifier 10.1109/TNET.2012.2183643 likely to have long-term characteristics and are less volatile than node mobility, social-based forwarding schemes [10], [20] outperform traditional schemes that are based on oblivious heuristic [37] or mobility-based prediction [26], [42].

Most data forwarding schemes focus on forwarding data to a single destination. Multicast, on the other hand, is more effective for data dissemination and multiparty communication. For example, in sparse vehicular ad hoc networks (VANETs), a vehicle may disseminate live traffic information to other following vehicles. In extreme scenarios such as battlefields, where cellular connectivity may be unavailable, multiple mobile users may share information with each other to allow collaborative decisions. However, effectively supporting multicast is challenging in DTNs. Although there are some initial research efforts on multicasting in DTNs, they are limited to semantic multicast models [43] and multicast capacity analysis [24]. Some others introduced social community for multicast [7], but did not provide any analytical model or strategy for selecting relays from a social network perspective.

In this paper, we propose a probabilistic approach to socialaware multicast in DTNs. We exploit social network concepts such as centrality and community to improve the cost-effectiveness of multicast. More specifically, our approach multicasts data to the destinations with the required forwarding probability and time constraint while minimizing the data forwarding cost measured by the number of relays used. From this probabilistic perspective, the essential difference between multicast and unicast in DTNs is that the selection of relays for multicast is based on the relays' cumulative probabilities of forwarding data to multiple destinations.

We first consider multicasting a single data item in the network, and then generalize the problem to multiple data items with the constraint of limited relay buffer. Our detailed contributions are twofold. First, we propose effective relay selection strategies for both single-data and multiple-data multicast problems based on social network concepts. In particular, we provide community-based solutions for effectively maintaining global network knowledge at individual mobile nodes. Such knowledge is critical for calculating the cumulative data forwarding probability to multiple destinations. Second, we develop analytical models for multicast relay selection, and furthermore derive theoretical performance bounds of our multicasting schemes. These theoretical results show that the proposed strategies are able to select better nodes as relays and are able to ensure that the performance requirements for multicasting can be achieved in various network situations.

The rest of this paper is organized as follows. Section II provides an overview of the problem definitions, the basic idea, and the weighted network modeling. Based on this modeling, single-data and multiple-data multicast problems are studied in 
Sections III and IV, respectively. Section V evaluates the performance of our approach, Section VI reviews existing work, and Section VII concludes the paper.

\section{OVERVIEW}

\section{A. Problem Definitions and Assumptions}

Our initial focus is on multicasting a single data item.

Problem 1: Single-Data Multicast (SDM)

$\{p, \mathbb{D}, T\}$ : To deliver a data item to a set $\mathbb{D}$ of destinations, how should one choose the minimum number of relays to achieve the delivery ratio $p$ within the time constraint $T$ ?

The SDM Problem is then generalized as follows.

Problem 2: Multiple-Data Multicast (MDM)

$\left\{p, \mathbb{D}_{1}, \ldots, \mathbb{D}_{n}, s_{1}, \ldots, s_{n}, T\right\}$ : To deliver a set of data items $d_{1}, d_{2}, \ldots, d_{n}$ with sizes $s_{1}, \ldots, s_{n}$, from a data source to destination sets $\mathbb{D}_{1}, \mathbb{D}_{2}, \ldots, \mathbb{D}_{n}$, what is the method for choosing the minimum number of relays to achieve the delivery ratio $p$ within the time constraint $T$ ?

In these problems, delivery ratio $p$ is, generally, the ratio of data items being delivered to the destinations within the time constraint $T$. In SDM, $p$ is the portion of destinations having received data within $T$. In MDM, $p$ is generalized to be $\frac{1}{n} \sum_{i=1}^{n}\left(k_{i} /\left|\mathbb{D}_{i}\right|\right)$, where $k_{i}$ is the number of nodes in $\mathbb{D}_{i}$ that receive data $d_{i}$ within $T$. In particular, for a specific destination, its delivery ratio is equivalent to the probability that it receives data within $T$. We assume that each node in DTNs has only limited buffer for storing and forwarding data.

\section{B. Big Picture}

Our basic idea is to develop social-based metrics for relay selection, allowing the metrics to evaluate a node's capability for forwarding data to its destinations. Our relay selection strategy then aims at selecting the minimum number of relays to satisfy the performance requirements of multicasting in terms of delivery ratio and delay. ${ }^{1}$

We propose a centrality-based heuristic for SDM based on a probabilistic centrality metric. The destinations are assumed to be randomly distributed in the network, and hence the centrality metric of a node measures the node's capability of forwarding data to each destination. Then, the data source does not distinguish the data forwarding probabilities to different destinations for relay selection, and centrality metrics of relays can be calculated based on the local knowledge of the data source about its contacted neighbors. ${ }^{2}$ If a relay does not have sufficient buffer for storing the data, the data source simply selects another relay, and consequently the consideration of node buffer constraint in relay selection becomes trivial.

Relay selection for MDM is more challenging because the relays should be aware of their probabilities of forwarding each data item to its destinations. This capability is called "destination-awareness" throughout this paper, and the necessity of this capability stands as the essential difference between SDM and MDM. This capability is required for MDM mainly due to the limited node buffer in DTNs. For example, as shown in Fig. 1, the data source $S$ multicasts two data items $d_{1}, d_{2}$ to the destination sets $\mathbb{D}_{1}, \mathbb{D}_{2}$, respectively, but relay $R_{1}$ can only carry one

\footnotetext{
${ }^{1}$ In this paper, the delay of data delivery is measured as the average time for data being transmitted to its destinations after it is generated.

${ }^{2}$ The nodes that have been contacted by the data source.
}

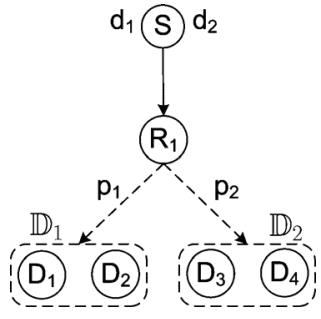

Fig. 1. Necessity of destination-awareness.

data item. To improve the delivery ratio, $R_{1}$ should carry $d_{1}$ if its probability $p_{1}$ of forwarding $d_{1}$ to the destinations in $\mathbb{D}_{1}$ is higher than $p_{2}$. Otherwise, $d_{2}$ should be carried by $R_{1}$. Thus, $S$ must have destination-awareness, which requires global knowledge about the social relation between the destinations and other nodes in the network. We propose a community-based approach to MDM relay selection, which only requires a node to maintain its destination-awareness within its local community and effectively reduces the overhead of maintaining global knowledge in DTNs.

The SDM and MDM definitions consider that all data items are generated by the same source node. When data items originate from multiple data sources simultaneously, selection of relays can be similar to that in the MDM problem. More specifically, when a node is selected as the relay by multiple data sources simultaneously, it determines which data items to carry based on its destination-awareness and the consequent probabilities of forwarding data to the destinations.

\section{Network Modeling}

Node contacts, as well as the social relations among nodes in DTNs, are described by the network contact graph $G(V, E)$, where the stochastic contact process between each pair of nodes $N_{i}, N_{j} \in V$ is modeled as an edge $e_{i j} \in E$. We assume that node contacts are symmetric; i.e., $N_{j}$ contacts $N_{i}$ whenever $N_{i}$ contacts $N_{j}$, and $G$ is therefore undirected.

The characteristics of an edge $e_{i j} \in E$ are mainly determined by the properties of intercontact time among mobile nodes, which have been studied intensively. From a theoretical perspective, [5] has proved that the pairwise intercontact time is exponentially distributed in representative mobility models such as Random Way Point (RWP). From an experimental perspective, [23] has validated that the aggregate distribution of the intercontact time over all node pairs is a mixture of power-law and exponential distributions.

Comparatively, modeling the characteristics of the distribution of pairwise intercontact time is more difficult due to the heterogeneity of contact behaviors among different nodes. As an initial effort, [8] examined various forms of distributions, among which log-normal distribution provides the best fitting accuracy to the pairwise intercontact time of heterogeneous human mobility, and the accuracy of exponential or power-law distributions is also acceptable. In contrast, later study [44] of vehicular mobility draws an opposite conclusion to support the distribution of pairwise intercontact time to be exponential.

In general, there is still no conclusive agreement on the distribution of pairwise intercontact time. In this paper, we follow the existing forwarding schemes [1] and analytical studies [22], [24] to consider such distribution as exponential because this model has been shown in literature to provide 
TABLE I

TRACE SUMMARY

\begin{tabular}{c|ccc}
\hline Trace & Infocom & MIT Reality & UCSD \\
\hline \hline Network type & Bluetooth & Bluetooth & WiFi \\
\# devices & 41 & 97 & 275 \\
\# internal contacts & 22,459 & 114,046 & 123,225 \\
Duration (days) & 3 & 246 & 77 \\
Pairwise contact & 4.6 & 0.049 & 0.021 \\
freq. (per day) & & & \\
\hline
\end{tabular}

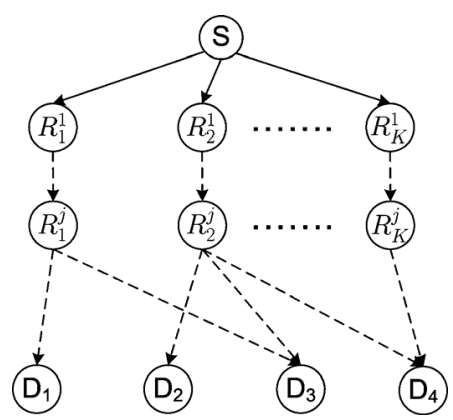

Fig. 2. SDM data forwarding process.

a satisfactory level of fitting accuracy for relay selection decisions. The contacts between nodes $N_{i}$ and $N_{j}$ then form a homogeneous Poisson process characterized by the pairwise contact rate $\lambda_{i j}$. This rate is calculated from the cumulative contacts between nodes $N_{i}$ and $N_{j}$ since the network starts and gains dynamic updating from these two nodes themselves.

\section{Experimental Traces}

We use three sets of realistic DTN traces to evaluate the performance of our multicast schemes. These traces record contacts among users carrying mobile devices in mobile network environments including university campuses (Infocom [6] and MIT Reality [11]) or conference sites (UCSD [28]). The devices are equipped with Bluetooth or Wi-Fi interfaces to detect and communicate with each other. In Infocom and MIT Reality traces, the devices periodically detect their peers via their Bluetooth interfaces, and a contact is recorded when two devices move close to each other. In the UCSD trace that consists of Wi-Fi-enabled devices, the devices search for nearby Wi-Fi access points (APs) and associate themselves to the APs with the best signal strength. A contact is recorded when two devices are associated to the same AP. As summarized in Table I, these traces differ in their scales and durations.

\section{Single-Data Multicast}

In this section, we develop a centrality-based heuristic for SDM based on the local knowledge of the data source about the centrality of its contacted neighbors. As shown in Fig. 2, we assume that the destinations are randomly distributed in the network, and the data source $S$ initially selects a minimum number $(K)$ of relays that ensure that the required delivery ratio $p$ can be achieved within the time constraint $T$. Each relay $R_{i}^{j}$ then only forwards the data to another node $R_{i}^{j+1}$ whose centrality metric is higher than that of $R_{i}^{j}$, and $R_{i}^{j}$ removes its local data copy afterwards. Therefore, the number of relays in the network remains $K$. Similar relay selection

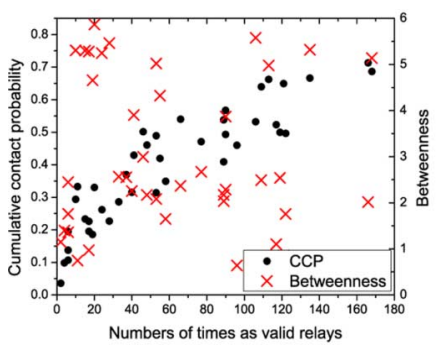

(a)

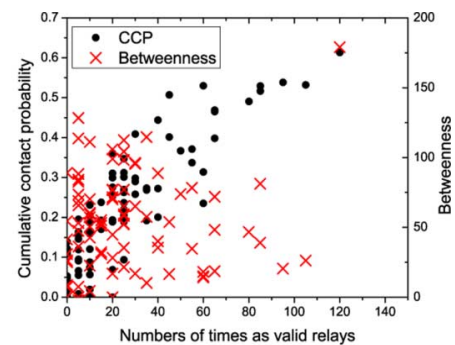

(b)
Fig. 3. Node centrality values and the number of times for them to be counted as valid relays. (a) Infocom. (b) MIT Reality.

strategy has also been used in Spray-and-Wait [34]. However, the strategy in Spray-and-Wait assumes that the contact patterns of mobile nodes are homogeneous and each relay has equal capability of forwarding data to the destinations. Hence, this strategy may not operate well in practice.

\section{A. Centrality Metric}

We propose our centrality metric as follows.

Definition 1: Cumulative Contact Probability (CCP): The CCP of a node $N_{i}$ is defined as

$$
C_{i}=1-\frac{1}{N-1} \sum_{j=1, j \neq i}^{N} e^{-\lambda_{i j} T}
$$

where $N$ is the number of nodes in the network, and $\lambda_{i j}$ is the contact rate between node $N_{i}$ and $N_{j}$. According to our network modeling in Section II-C, $1-e^{-\lambda_{i j} T}$ is the probability that node $N_{i}$ contacts $N_{j}$ within time $T$. Consequently, $C_{i}$ indicates the expected probability that a random node in the network has been contacted by $N_{i}$ within time $T$.

Compared to the betweenness centrality metric [14] that is widely used for social-aware data forwarding [10], [20] and measures the importance of a node's facilitating communication among other nodes, CCP is more effective for modeling the relay selection process in DTNs. The reason is that betweenness is defined and calculated based on an unweighted network contact graph. Such a graph only evaluates the extent to which a node connects other nodes, but does not consider the different contact rates among mobile nodes. Hence, betweenness is incapable of accurately evaluating the capabilities for a node to contact other nodes in practice.

To better illustrate the advantage of CCP over betweenness, we run 20000 SDM scenarios with random data sources and destinations and use Epidemic routing [37] to forward data. We count a node as a "valid" relay if it is the first one to deliver data to a destination. In this way, the more times that a node is counted as a valid relay, the higher capability that this node has to contact the destinations. Considering that the destinations are randomly selected in the network, we believe that this number represents the capability of a node to facilitate the data forwarding process.

These numbers are shown in Fig. 3, where both betweenness and CCP centrality values of nodes are calculated. ${ }^{3}$ Fig. 3 shows that the $\mathrm{CCP}$ spots are more likely to form a straight line, which

\footnotetext{
${ }^{3}$ Betweenness of nodes is calculated in an ego-centric manner [27]
} 
TABLE II

NUMERIC RESUlTS OF LEAST-SQUARE FITTING

\begin{tabular}{c|c|c|c}
\hline Metric & & CCP & betweenness \\
\hline \hline \multirow{3}{*}{ Infocom } & $a$ & 0.00339 & -0.00054 \\
& $b$ & 0.189 & 3.0642 \\
& $e$ & 0.7526 & 35.994 \\
\hline \multirow{3}{*}{ MIT Reality } & $a$ & 0.0053 & 0.271 \\
& $b$ & 0.0804 & 42.954 \\
& $e$ & 3.4159 & 2398.9 \\
\hline
\end{tabular}

indicates that a node with higher $\mathrm{CCP}$ is more effective in delivering data to the destinations. Comparatively, the scattered betweenness spots represent higher randomness, which indicates that nodes with higher betweenness may not necessarily contribute more to data forwarding. To quantify such difference, we conduct least-square linear curve fitting $(y=a x+b)$ for all CCP and betweenness spots $\left(x_{i}, y_{i}\right)$ in Fig. 3 , and our conclusion is supported by the normalized fitting error $e=\sum_{i=1}^{n} \frac{\left(y_{i}-\widetilde{y}_{i}\right)^{2}}{y_{i}}$ in Table II.

\section{B. Initial Relay Selection}

When the destinations are randomly distributed in the network, the general methodology of initial relay selection at the data source is to ensure that each node in the network has a probability higher than $p$ to be contacted by the selected relays within $T$. As a prerequisite, we assume that the destinations are uniformly distributed in the network, so that each node other than the data source has an equal chance to be a destination.

1) Selecting Nodes in Contact: If the data source $S$ is in contact with a set of nodes $\mathcal{R}=\left\{R_{1}, R_{2}, \ldots, R_{k}\right\}$ that can be selected as relays, then the selected relays should contact the remaining $N-k-1$ nodes in the network to ensure that all destinations are contacted by the relays. We assume that none of the destinations is in the set $\mathcal{R}$, otherwise $S$ can trivially forward data to the destinations immediately.

When we randomly choose a node $N_{j} \notin \widetilde{\mathcal{R}}$, where $\tilde{\mathcal{R}}=$ $\{S\} \cup \mathcal{R}$, the node $R_{i} \in \mathcal{R}$ has probability $1-e^{-\lambda_{i j} T}$ to contact $N_{j}$ within time $T$, where $\lambda_{i j}$ is the contact rate between $R_{i}$ and $N_{j}$. Hence, the expected probability that a random node $N_{j} \notin \widetilde{\mathcal{R}}$ is not contacted by $R_{i}$ within time $T$ is

$$
\begin{aligned}
p_{i} & =\frac{1}{N-k-1} \cdot \sum_{j=1, j \notin \tilde{\mathcal{R}}}^{N} e^{-\lambda_{i j} T} \\
& =\frac{N-1}{N-k-1} \cdot\left(1-C_{i}\right)-\frac{1}{N-k-1} \cdot \sum_{j \in \tilde{\mathcal{R}}, j \neq i} e^{-\lambda_{i j} T} .
\end{aligned}
$$

Every $R_{i} \in \mathcal{R}$ locally calculates and sends its $p_{i}$ to $S$ for relay selection. To ensure that the average delivery ratio is higher than $p$, the probability that a random node $N_{j} \notin \tilde{\mathcal{R}}$ is not contacted by the relays should be lower than $1-p$, i.e.,

$$
\prod_{i=1}^{k}{p_{i}}^{x_{i}} \leq 1-p
$$

where $x_{i} \in\{0,1\}$ indicates whether or not $R_{i}$ is selected as relay.

2) Selecting Nodes Not in Contact: When $S$ selects relays, some of its contacted neighbors with higher centrality may not be in contact with $S$. To select relays among these nodes, we should also consider the elapsed time for them to contact $S$.

For a contacted neighbor $R_{i}$ of $S$, if the random variable $T_{1}$ is the time for $S$ to contact $R_{i}$, and $T_{2}$ is the time for $R_{i}$ to contact another node $N_{j}$, then the probability that $S$ can forward data to $N_{j}$ via $R_{i}$ within time $T$ is $\mathbb{P}\left(T_{1}+T_{2} \leq T\right)$, which is calculated as follows:

$$
\begin{aligned}
p_{i j} & =\mathbb{P}\left(T_{1}+T_{2} \leq T\right)=\int_{0}^{T} f_{1}(t) \otimes f_{2}(t) d t \\
& =\int_{0}^{T} d t\left(\int_{0}^{t} f_{1}(\tau) f_{2}(t-\tau) d \tau\right) \\
& =1-\frac{\lambda_{i j} e^{-\lambda_{S i} T}-\lambda_{S i} e^{-\lambda_{i j} T}}{\lambda_{S i}-\lambda_{i j}}
\end{aligned}
$$

where $\otimes$ indicates convolution of functions, and $f_{1}(t)$ and $f_{2}(t)$ are the probability density functions (PDFs) of $T_{1}$ and $T_{2}$, respectively.

The expected probability that $S$ cannot forward data to a random node $N_{j} \notin \widetilde{\mathcal{R}}$ via $R_{i}$ within time $T$ is

$$
\widetilde{p}_{i}=\frac{1}{N-k-1} \sum_{j=1, j \notin \widetilde{\mathcal{R}}}^{N}\left(1-p_{i j}\right)
$$

and $S$ then selects relays in the similar way as described in (2), using $\widetilde{p}_{i}$ instead of $p_{i}$.

However, in practice it is difficult for $S$ to do the calculation in (3) because it requires $S$ to know the contact rate $\lambda_{i j}$ between nodes $R_{i}$ and $N_{j}$. Instead, we exploit the following lower bound as an approximation to $p_{i j}$ in (3).

Theorem 1: For any fixed $\widetilde{T} \in(0, T)$, we have

$$
p_{i j}=\mathbb{P}\left(T_{1}+T_{2} \leq T\right) \geq\left(1-e^{-\lambda_{S i} \widetilde{T}}\right)\left(1-e^{-\lambda_{i j}(T-\widetilde{T})}\right) .
$$

Proof: The right-hand side (r.h.s.) of (5) indicates the probability

$$
\mathbb{P}\left(T_{1} \leq \widetilde{T}\right) \cdot \mathbb{P}\left(T_{2} \leq T-\widetilde{T}\right) .
$$

We define two sets $\mathbb{T}_{1}$ and $\mathbb{T}_{2}$ as

$$
\left\{\begin{array}{l}
\mathbb{T}_{1}=\left\{\left(T_{1}, T_{2}\right) \mid T_{1}+T_{2} \leq T\right\} \\
\mathbb{T}_{2}=\left\{\left(T_{1}, T_{2}\right) \mid T_{1} \leq \widetilde{T}, T_{2} \leq T-\widetilde{T}\right\} .
\end{array}\right.
$$

It is obvious that for any element $\left\{t_{1}, t_{2}\right\} \in \mathbb{T}_{2}$, we also have $\left\{t_{1}, t_{2}\right\} \in \mathbb{T}_{1}$, which means $\mathbb{T}_{2} \subseteq \mathbb{T}_{1}$. Therefore

$$
\mathbb{P}\left(T_{1}+T_{2} \leq T\right) \geq \mathbb{P}\left(T_{1} \leq \widetilde{T}\right) \cdot \mathbb{P}\left(T_{2} \leq T-\widetilde{T}\right)
$$

which proves the theorem.

The tightest bound in (5) is achieved by $\widetilde{T}_{0}$ when $\left.\frac{\partial p_{i j}}{\partial \widetilde{T}}\right|_{\widetilde{T}}=\widetilde{T}_{0}=0$, and this equation has only one solution in $(0, T)$. In practice, each contacted neighbor $R_{i}$ of $S$ numerically calculates the optimal $\widetilde{T}_{0}$ for $N_{j}$ using Newton's method, and then calculates

$$
\widetilde{p}_{i j}=\left(1-e^{-\lambda_{S i} \widetilde{T}_{0}}\right)\left(1-e^{-\lambda_{i j}\left(T-\widetilde{T}_{0}\right)}\right)
$$

as a lower bound of $p_{i j}$ in (3). $\widetilde{p}_{i j}$ is then used by $S$ in (4) to calculate $\widetilde{p}_{i}$ for relay selection. 


\section{Discussions}

Our proposed scheme implicitly assumes that the pairwise contact rate $\lambda_{i j}$ between nodes $i$ and $j$ remains stationary over time, and estimates the probability for node $i$ to contact node $j$ within time $T$ in the future as $p_{i j}=1-e^{-\lambda_{i j} T}$. In practice, if the contact rate between nodes $i$ and $j$ during time $T$ in the future is $(1+\Delta) \lambda_{i j}$, our estimation has the following error:

$$
\Delta p_{i j}=e^{-\lambda_{i j} T} \cdot\left(1-e^{-\Delta \cdot \lambda_{i j} T}\right) \leq e^{-\lambda_{i j} T} \cdot p_{i j}
$$

According to Table I, usually we have $\lambda_{i j} \ll 1$, and hence such estimation error is very limited. Determining analytically the impact of such error on the multicasting performance is difficult because $\Delta p_{i j}$ can be either positive or negative. Instead, in Section V-D, we experimentally investigate this impact via trace-driven simulations in particular network scenarios.

\section{Multiple-Data Multicast}

\section{A. Overview}

In this section, we propose a community-based approach to MDM. We assume that the network consists of a number of social communities, and each node belongs to at least one community. The community structure in the network is identified by a distributed community detection scheme [21]. Gateway nodes, which belong to multiple communities simultaneously, connect these communities.

Due to limited node buffer in DTNs, destination-awareness is maintained at each individual node. As a result, a relay can accurately estimate the probability for it to forward a specific data item to its destinations, and hence choose the most appropriate data items to carry. A node $A$ maintains its destination-awareness of another node $B$ in the form of an opportunistic path defined as follows.

Definition 2 Opportunistic Path: An n-hop opportunistic path $P_{A B}=\left(V_{P}, E_{P}\right)$ between two nodes $A$ and $B$ consists of a node set $V_{P}=\left\{A, N_{1}, N_{2}, \ldots, N_{n-1}, B\right\}$ and an edge sequence $E_{P}=\left\{e_{1}, e_{2}, \ldots, e_{n}\right\}$ with edge weights $\left\{\lambda_{1}, \lambda_{2}, \ldots, \lambda_{n}\right\}$. Each edge corresponds to an opportunistic contact process on the network contact graph.

To reduce the overhead of maintaining destination-awareness, the data source maintains its destination-awareness for nodes in the same community at a fine-grained level, and for nodes in other communities at a coarse-grained level. Fine-grained destination-awareness includes the complete opportunistic path to a node, i.e., the complete information about $V_{P}, E_{P}$ and the edge weights in Definition 2. Coarse-grained destination-awareness only includes the gateway nodes connecting different communities on the opportunistic path, i.e., a subset of $V_{P}$, and the weights of opportunistic paths connecting these gateway nodes. Such coarse-grained destination-awareness is called "gateway path" throughout the rest of this section, and the difference between opportunistic path and gateway path is illustrated in Fig. 4.

Correspondingly, multicast is conducted in a hierarchical manner as follows.

- Intracommunity multicast forwards data to the destinations in the same community with the data source. The data source selects relays among its contacted neighbors based on the maintained fine-grained destination-awareness.

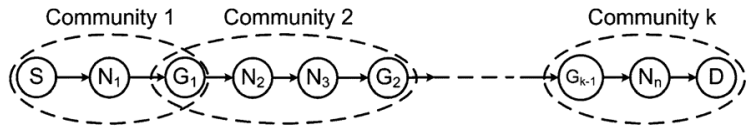

(a)

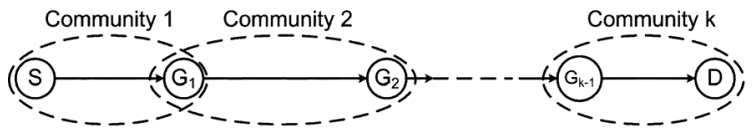

(b)

Fig. 4. Maintenance of destination-awareness with different granularities. (a) Fine-grained: opportunistic path. (b) Coarse-grained: gateway path.

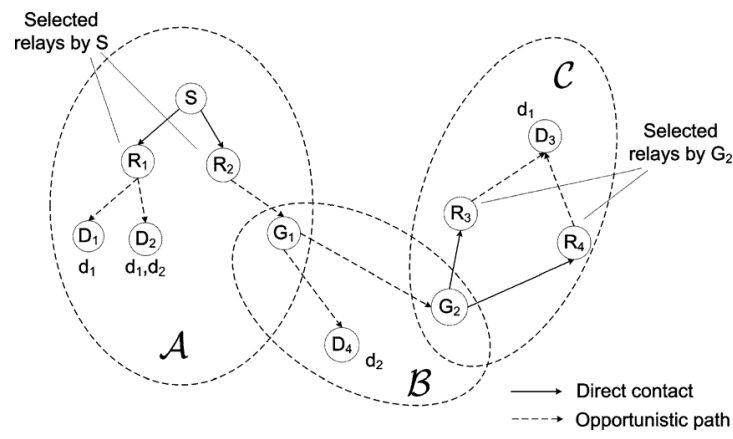

Fig. 5. MDM data forwarding process.

Each relay then forwards data to a destination according to the opportunistic path to that destination.

- Intercommunity multicast forwards data to other destinations in different communities. It is divided into multiple stages of intracommunity multicast, each of which corresponds to a gateway node on the maintained gateway path from the data source to the destinations. In each stage, the gateway node acts as the data source within the corresponding community and selects relays for forwarding data to the next gateway node.

This hierarchical multicasting process is illustrated in Fig. 5, where $S$ multicasts two data items $d_{1}$ and $d_{2}$ to destination sets $\left\{D_{1}, D_{2}, D_{3}\right\}$ and $\left\{D_{2}, D_{4}\right\}$, respectively. For destinations $D_{1}$ and $D_{2}$ in the same community with $S, R_{1}$ is selected by $S$ as the relay, and $R_{1}$ forwards $d_{1}$ and $d_{2}$ to the destinations according to its recorded opportunistic paths to $D_{1}$ and $D_{2}$.

For destination $D_{3}$ that resides in another community, $S$ knows that reaching $D_{3}$ is via the gateway nodes $G_{1}$ and $G_{2}$, although $S$ does not record the detailed opportunistic paths in communities $\mathcal{B}$ and $\mathcal{C}$. The multicast process then consists of three stages corresponding to the communities $\mathcal{A}, \mathcal{B}$, and $\mathcal{C}$, connected by $G_{1}$ and $G_{2}$. In community $\mathcal{A}, S$ only selects relays to forward $d_{1}$ to $G_{1}$. In community $\mathcal{B}, G_{1}$ acts as the data source and selects relays to forward $d_{1}$ to the next gateway node $G_{2}$. In community $\mathcal{C}, G_{2}$ acts as the data source and selects relays to send $d_{1}$ to $D_{3}$.

\section{B. Weight of Opportunistic Path}

The weight of an opportunistic path between nodes $A$ and $B$ is the probability $p_{A B}(T)$ that data is forwarded from $A$ to $B$ along $P_{A B}$ within time $T$. According to Definition 2 , the intercontact time $X_{i}$ between nodes $N_{i}$ and $N_{i+1}$ follows an exponential distribution with PDF $p_{X_{i}}(x)=\lambda_{i} e^{-\lambda_{i} x}(x \geq 0)$, and the total time for transferring data from $A$ to $B$ along $P_{A B}$ 


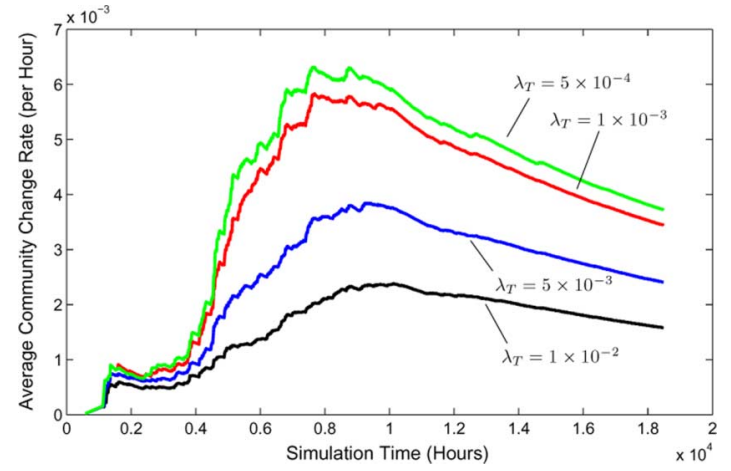

Fig. 6. Rate of community changes.

is $Y=\sum_{i=1}^{n} X_{i}$. The PDF $p_{Y}(x)$ is calculated by convolutions on $p_{i}(x)$ as

$$
p_{Y}(x)=p_{1}(x) \otimes p_{2}(x) \cdots \otimes p_{n}(x) .
$$

Theorem 2: ([33, Section 5.2.4]) For an $n$-hop opportunistic path with edge weights $\lambda_{1}, \ldots, \lambda_{n}, p_{Y}(x)$ is expressed as

$$
p_{Y}(x)=\sum_{i=1}^{n} C_{i}^{(n)} p_{X_{i}}(x)
$$

where $p_{X_{i}}(x)=\lambda_{i} e^{-\lambda_{i} x}$, and $C_{i}^{(n)}=\prod_{j=1, j \neq i}^{n} \frac{\lambda_{j}}{\lambda_{j}-\lambda_{i}}$.

From $p_{Y}(x)$, the weight of opportunistic path $P_{A B}$ is

$$
\begin{aligned}
p_{A B}(T) & =\mathbb{P}(Y<T)=\int_{0}^{T} p_{Y}(x) d x \\
& =\sum_{i=1}^{n} C_{i}^{(n)} \cdot\left(1-e^{-\lambda_{i} T}\right) .
\end{aligned}
$$

\section{Maintenance of Destination-Awareness}

In our approach, we use the $k$-clique community detection scheme [31] to detect overlapping communities and gateway nodes. According to Palla et al. [31], a $k$-clique community is a union of all $k$-cliques (complete subgraphs of size $k$ ) that can reach each other through a series of adjacent $k$-cliques. In the distributed implementation of the $k$-clique scheme for DTNs [21], each node first builds a familiar set containing its contacted neighbors based on specific admission criterion, and then builds its local community by merging the familiar sets of other nodes. The number of gateway nodes is controlled by $k$ as well as the threshold for determining the familiar set. We adopt the $k$-clique scheme using the pairwise contact rate as the admission criterion for familiar sets.

The accuracy of maintaining destination-awareness is related with the changes of social community structures in the network. We define a community change as an operation of adding a node to or deleting a node from a community. The rate of community change is hence defined as the number of such operations conducted per hour. We investigate the rate of community change on the MIT Reality trace. The results in Fig. 6 show that this rate is lower than $7 \times 10^{-3}$ per hour. This low rate thereby ensures maintaining accurate and up-to-date destination-awareness at individual nodes.

1) Intracommunity Destination-Awareness: For nodes in the same community, a node maintains its intracommunity destina-
TABLE III RECORD OF OPPORTUNISTIC PATH

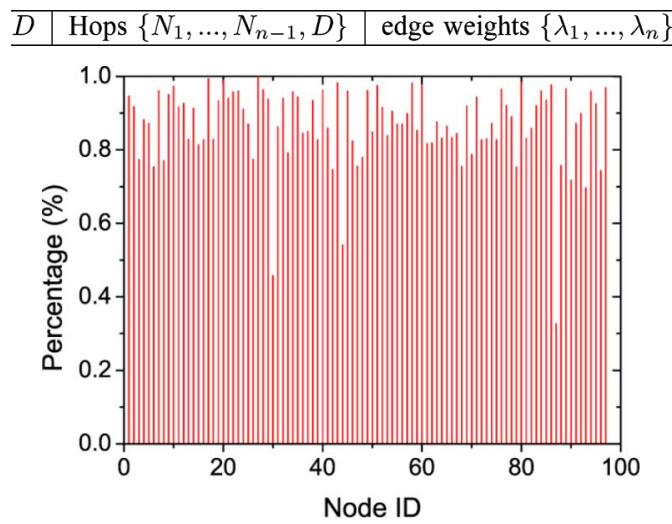

Fig. 7. Intracommunity destination-awareness.

tion-awareness as an opportunistic path table. The record format for the opportunistic path to a node $D$ is shown in Table III. If a node belongs to multiple communities, a separate table is maintained for each community. Initially, each node only has the information about its contacted neighbors in the table. When a node $A$ contacts another node $B$ within the same community, they exchange and update their tables. For a record of node $D$ in $B$ 's opportunistic path table, if no record exists for $D$ at $A, A$ adds this record into its own table. Otherwise, if the path from $A$ to $D$ via $B$ has larger weight than the path recorded by $A, A$ updates its local record for $D$ using $B$ 's record for $D$.

A node $A$ is aware of another node $B$ if it has the information about the opportunistic path to $B$. Furthermore, we quantify the intracommunity destination-awareness using the percentage of nodes of which a node is aware within its local community. To investigate intracommunity destination-awareness in practice, we conduct distributed $k$-clique community detection described in [21] on MIT Reality trace and allow nodes to maintain and exchange their opportunistic path tables during $20 \%$ of the trace length. As shown in Fig. 7, every node is aware of most of the other nodes within its local community. This result supports our approach to intracommunity multicast, which is based on the maintained opportunistic paths among mobile nodes.

2) Intercommunity Destination-Awareness: Intercommunity destination-awareness among nodes in different communities is maintained in the form of gateway paths. For example, in Fig. 5, $S$ maintains the destination-awareness to $D_{3}$ as $S \rightarrow G_{1} \rightarrow$ $G_{2} \rightarrow D_{3}$, which indicates that the data transits through three communities from $S$ to $D_{3}$. To accomplish this, $G_{1}$ and $G_{2}$ exchange their maintained information about nodes in communities $\mathcal{A}$ and $\mathcal{C}$ when they contact. $S$ then knows the gateway path to $D_{3}$ when it contacts $G_{1}$.

Clearly, reduction of the overhead of maintaining intercommunity destination-awareness results from using gateway paths, as illustrated in Fig. 4 . When there are $N$ nodes in the network that form $K$ communities, such overhead is reduced by $O\left(\sqrt{\frac{N}{K}}\right)$ times compared to maintaining the complete opportunistic path. According to the results reported in [20], realistic DTN traces usually have $N \gg K$. For example, in MIT Reality trace when $k$-clique community detection is used and $k=4$, we have $N=97$ and $K=5$. The exploitation of gateway paths then reduces the overhead of maintaining intercommunity des- 
tination-awareness by up to 4.4 times. This reduction generally increases when $k$ becomes larger or the admission threshold for familiar sets during community detection becomes lower.

\section{Intracommunity Multicast}

In each community, the source selects relays according to the nodes' probabilities of forwarding data to the destinations in the same community. As illustrated in Fig. 5, the source and destinations in a community may be gateway nodes on the gateway paths from the data's original source to the final destinations.

We suppose that the source $S$ has data items $d_{1}, \ldots, d_{n}$ with sizes $s_{1}, \ldots, s_{n}$ and destination sets $\mathbb{D}_{1}, \ldots, \mathbb{D}_{n}$, and $S$ selects relays among nodes $R_{1}, \ldots, R_{m}$ with buffer sizes $B_{1}, \ldots, B_{m}$. Relay selection is formulated as follows:

$$
\begin{array}{ll}
\min & \left|\left\{j \mid \sum_{i=1}^{n} x_{i j}>0\right\}\right| \\
\text { s.t. } & \sum_{i=1}^{n} x_{i j} s_{i} \leq B_{j}, \quad \text { for } j=1, \ldots, m \\
& \frac{1}{\left|\mathbb{D}_{i}\right|} \sum_{k \in \mathbb{D}_{i}} \prod_{j=1}^{m}\left(1-x_{i j} p_{j k}\right) \leq(1-p), \quad \text { for } i=1, \ldots, n
\end{array}
$$

where $x_{i j} \in\{0,1\}$ indicates whether or not a data item $d_{i}$ is placed on relay $R_{j} . p_{j k}$ is the probability that data is forwarded to destination $k$ via $R_{j}$ within time $T$ and is calculated from intracommunity destination-awareness. The second constraint ensures that, for each data item, its probability to be delivered to its destinations is higher than the required $p$.

The problem defined in (8) is NP-hard, and we instead propose a two-stage heuristic for relay selection. First, the optimal data items for each $R_{j}$ to carry are determined based on the node buffer constraint of $R_{j}$, to ensure that the data items carried by $R_{j}$ are the ones with the maximum probabilities to be forwarded to their destinations via $R_{j}$. Second, relays are selected using the optimized data forwarding probabilities calculated in the first stage.

1) Data Item Selection: The optimal data items for $R_{j}$ to carry can be determined by solving the following problem:

$$
\begin{aligned}
& \max \sum_{i=1}^{n} \sum_{k \in \mathbb{D}_{i}} \frac{x_{i j} p_{j k}}{\left|\mathbb{D}_{i}\right|} \\
& \text { s.t. } \quad \sum_{i=1}^{n} x_{i j} s_{i} \leq B_{j}
\end{aligned}
$$

where $\frac{1}{\left|\mathbb{D}_{i}\right|} \sum_{k \in \mathbb{D}_{i}} p_{j k}$ is the expected probability that data item $d_{i}$ is delivered to its destinations via $R_{j}$.

2) Relay Selection: According to the optimal data item selection on $R_{j}$, the weight $w_{j}$ for $R_{j}$ is

$$
w_{j}=1-\frac{1}{n} \sum_{i=1}^{n} \sum_{k \in \mathbb{D}_{i}} \frac{x_{i j} p_{j k}}{\left|\mathbb{D}_{i}\right|}
$$

which indicates the expected probability that a data item cannot be delivered to its destinations by $R_{j}$ within $T$. The stage of data item selection minimizes this probability.

Then, the node weight $w_{j}$ is used for relay selection, satisfying the following performance requirement:

$$
\prod_{j=1}^{m} w_{j}^{x_{j}} \leq 1-p
$$

(a)

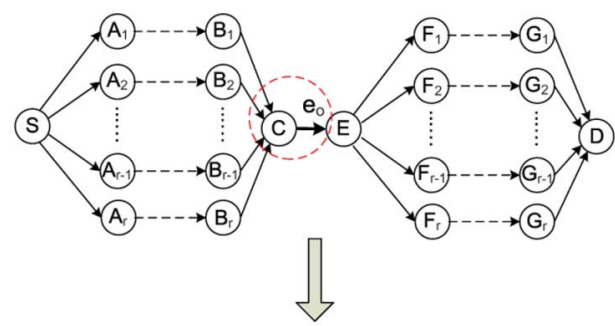

(b)

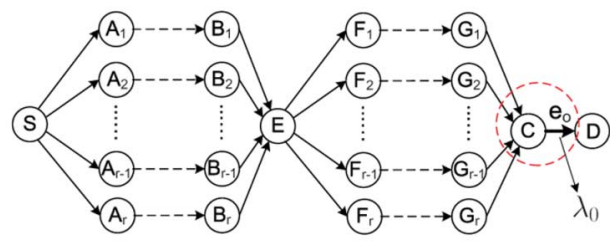

(c)

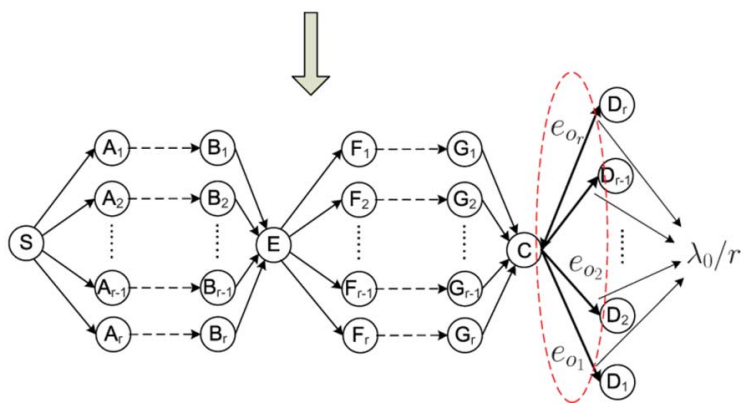

Fig. 8. Edge splitting process.

where $x_{j} \in\{0,1\}$ indicates whether or not $R_{j}$ is a relay.

\section{E. Edge Splitting Process}

Section IV-D calculates the cumulative probability for multiple relays to forward a data item to the same destination. However, this calculation is usually difficult because the opportunistic paths from relays to the same destination may partially overlap, and the corresponding forwarding probabilities of different relays are not independent from each other. This situation negates the possibility of directly multiplying these probabilities together in (8) or (9). For example, in Fig. 8(a), $r$ paths from $S$ to $D$ via different relays $A_{1}, \ldots, A_{r}$ share the edge $e_{0}=(C, E)$ in common. ${ }^{4}$

In order to calculate such cumulative data forwarding probabilities, this section proposes an edge splitting process to eliminate the overlap among opportunistic paths. First, the commutativity of convolution allows "moving" $e_{0}$ to the "end" of the paths, i.e., the last hop to the destination, without changing the weight of any involved path. This is illustrated in Fig. 8(b). Since a node contacts each of its neighbors independently, node $E$ shared by multiple paths does not affect the independence of the paths.

Thereafter, as in Fig. 8(b), if $T_{1}$ is the time for $S$ to send data to $C$ with PDF $f(t, r)$, and $T_{2}$ is the time for $C$ to contact $D$ with PDF $g\left(t, \lambda_{0}\right)=\lambda_{0} e^{-\lambda_{0} T}$, we have

$$
\mathbb{P}\left(T_{1}+T_{2} \leq T\right)=P_{t}(T, r)=\int_{0}^{T} f(t, r) \otimes g\left(t, \lambda_{0}\right) d t
$$

where

$$
f(t, r)=\frac{\partial}{\partial t}\left(1-\prod_{i=1}^{r}\left(1-\int_{0}^{t} f_{i}(u) d u\right)\right)
$$

\footnotetext{
${ }^{4}$ The dashed lines in Fig. 8(a) indicate opportunistic paths.
} 
and $f_{i}(t)$ is the PDF of the time for $S$ to send data to $C$ via the $i$ th path. We then have the following lemma.

Lemma 1: For $\forall T>0, r \geq 1, P_{t}(T, r) \leq P_{t}(T, r+1)$.

Proof: An intuitive proof of Lemma 1 can be as follows. When the number of paths from $S$ to $C$ increases from $r$ to $r+1$, the newly added $(r+1)$ th path generally reduces the time required for $S$ to send data to $C$ from a probabilistic perspective, and therefore increases the probability that $S$ can send data to $D$ within $T$.

However, difficulty arises for the data source $S$ to calculate $\mathbb{P}\left(T_{1}+T_{2} \leq T\right)$ from (10) and (11) because deriving the analytical form of $f(t)$ in (11) and its convolution with $g\left(t, \lambda_{0}\right)$ is difficult. Therefore, we exploit an edge splitting process to provide a lower bound on $\mathbb{P}\left(T_{1}+T_{2} \leq T\right)$, which is much easier to calculate in practice.

This edge splitting process is illustrated in Fig. 8(c). For an edge $e_{0}$ with rate $\lambda_{0}$ being shared by $r$ paths, we split $e_{0}$ to $r$ distinct edges, each of which has rate $\lambda_{0} / r$, and each of the $r$ paths is allocated a split edge. This process is equivalent to creating a virtual destination node for each of the $r$ paths. After the edge splitting process, if $T_{2}$ is the first time that $C$ contacts a virtual destination among $D_{i}$, we have

$$
\begin{aligned}
\mathbb{P}\left(T_{1}+T_{2} \leq T\right) & =P_{s}(T, r) \\
& =1-\prod_{i=1}^{r}\left(1-\int_{0}^{T} f_{i}(t) \otimes g\left(t, \frac{\lambda_{0}}{r}\right) d t\right) .
\end{aligned}
$$

Since the weight of the $i$ th path after edge splitting is calculated by (7), we can easily calculate $P_{s}(T, r)$ for any $T$. By comparing $P_{t}(T, r)$ and $P_{s}(T, r)$, we have the following.

Theorem 3: For $\forall T>0, r \geq 1, P_{s}(T, r) \leq P_{t}(T, r)$.

Proof: We prove this theorem by induction over $r$. First

$$
P_{s}(T, 1)=P_{t}(T, 1)=\int_{0}^{T} f_{1}(t) \otimes g\left(t, \lambda_{0}\right) d t .
$$

If $P_{s}(T, r) \leq P_{t}(T, r)$ for $r \geq 1$, from (12) we have

$$
\begin{aligned}
P_{s} & (T, r+1)-P_{s}(T, r) \\
& =1-P_{s}(T, r)-\prod_{i=1}^{r+1}\left(1-\int_{0}^{T} f_{i}(t) \otimes g\left(t, \frac{\lambda_{0}}{r+1}\right) d t\right) \\
& \leq 1-P_{s}(T, r)-\prod_{i=1}^{r+1}\left(1-\int_{0}^{T} f_{i}(t) \otimes g\left(t, \frac{\lambda_{0}}{r}\right) d t\right) \\
& =\left(1-P_{s}(T, r)\right) \cdot\left(1-\left(1-\int_{0}^{T} f_{r+1}(t) \otimes g\left(t, \frac{\lambda_{0}}{r}\right) d t\right)\right) \\
& \leq \frac{1}{r} \int_{0}^{T} f_{r+1}(t) \otimes g\left(t, \lambda_{0}\right) d t .
\end{aligned}
$$

On the other hand, (10) shows that $f(t, r+1)-f(t, r) \geq$ $\frac{1}{r} f(t, r+1)$. Then, from (10) and (11) we have

$$
\begin{aligned}
P_{t}(T, r+1)-P_{t}(T, r) & =\int_{0}^{T}(f(t, r+1)-f(t, r)) \otimes g\left(t, \lambda_{0}\right) d t \\
& \geq \frac{1}{r} \cdot \int_{0}^{T} f(t, r+1) \otimes g\left(t, \lambda_{0}\right) d t .
\end{aligned}
$$

By combining (13) and (14), we have $P_{s}(T, r+1)-$ $P_{s}(T, r) \leq P_{t}(T, r+1)-P_{t}(T, r)$. Given that $P_{s}(T, r) \leq$
$P_{t}(T, r)$, we can derive that $P_{s}(T, r+1) \leq P_{t}(T, r+1)$ for $\forall r \geq 1$. The theorem is therefore proved.

Theorem 3 shows that the edge splitting process provides a lower bound to the actual probability for the selected relays to forward data to a destination within the same community. Therefore, relay selection after the edge splitting process still guarantees that the performance requirements can be satisfied.

\section{F. Intercommunity Multicast}

To forward data according to a $k$-hop gateway path $S \rightarrow$ $G_{1} \rightarrow G_{2} \rightarrow \cdots \rightarrow G_{k-1} \rightarrow D$ to a destination $D$, the original data source $S$ simply divides the intercommunity multicast process into $k$ stages of intracommunity multicast, each of which corresponds to a distinct community on the gateway path from $S$ to $D$. In the $i$ th stage, the gateway node $G_{i-1}$ acts as the data source and selects relays within the $i$ th community to forward data to the next gateway node $G_{i}$.

The major challenge of intercommunity multicast is that $S$ should determine the parameters of performance requirements $p_{i}$ and $T_{i}$ for each stage of intracommunity multicast. In our approach, we fix $T_{i}=T / k$ and determine $p_{i}$ to ensure that the originally required delivery ratio $p$ can be achieved. According to Lemma $2, \prod_{i=1}^{k} p_{i} \geq p$ is a necessary requirement.

Lemma 2: If data needs time $T_{i}$ to be forwarded from $G_{i-1}$ to $G_{i}$ on the gateway path, $\mathbb{P}\left(\sum_{i=1}^{k} T_{i} \leq T\right) \geq \prod_{i=1}^{k} \mathbb{P}\left(T_{i} \leq \frac{T}{k}\right)$.

Proof: This lemma can be proved following the same method in the proof of Theorem 1 .

A straightforward solution is to set $p_{i}=p^{\frac{1}{k}}$ for $\forall i \in[1, k]$. However, there may exist $i \in[1, k]$, such that relays in the $i$ th community on the gateway path do not have sufficient contact capabilities to deliver data from $G_{i-1}$ to $G_{i}$ with probability $p^{\frac{1}{k}}$. In this case, $p_{i}$ is adjusted according to the specific data forwarding capabilities in individual communities.

Our basic idea is to maintain quantities $\left\{m p_{1}(T / k), m p_{2}(T / k), \ldots, m p_{k-1}(T / k)\right\}, \quad$ where $m p_{i}(T / k)$ is the maximal probability for forwarding data from $G_{i-1}$ to $G_{i}$ within time $T / k$. In practice, $m p_{i}(T / k)$ is calculated by the corresponding gateway node $G_{i-1}$, which maintains all opportunistic paths to the next gateway node $G_{i}$. If $G_{i-1}$ records $n_{i-1}$ opportunistic paths to $G_{i}$ with path weights $p_{1}(T / k), \ldots, p_{n_{i}}(T / k)$, we have $m p_{i}(T / k)=1-\prod_{j=1}^{n_{i-1}}\left(1-p_{j}(T / k)\right)$. As a result, $S$ determines $p_{i}$ as follows:

$$
p_{i}= \begin{cases}m p_{i}(T / k), & \text { if } m p_{i}(T / k) \leq p^{\frac{1}{k}} \\ \left(\frac{p}{p_{\text {low }}}\right)^{\frac{1}{k-k_{\text {low }}}}, & \text { else. }\end{cases}
$$

where $\mathcal{L}=\left\{i \mid m p_{i}(T / k) \leq p^{\frac{1}{k}}\right\}, k_{\text {low }}=|\mathcal{L}|$, and $p_{\text {low }}=$ $\frac{1}{k^{|\mathcal{L}|}} \prod_{i \in \mathcal{L}} m p_{i}(T / k)$.

In (15), if the maximally achievable delivery ratio $m p_{i}(T / k)$ in the $i$ th community is smaller than $p^{\frac{1}{k}}$, best-effort forwarding is exploited in the $i$ th community such that all contacted neighbors of $G_{i-1}$ are used as relays. Meanwhile, the probabilities $p_{i}$ for other communities increase accordingly, to ensure that $\prod_{i=1}^{k} p_{i} \geq p$ is satisfied.

Equation (15) and Lemma 2 lead to the following theorem for the performance of intercommunity multicast. 
Theorem 4: If the required delivery ratio $p$ is achievable in the network, our approach provides a solution to ensure that $p$ is achieved. Otherwise, our approach provides best-effort forwarding with a delivery ratio closest to $p$.

Proof: The proof of Theorem 4 consists of two cases:

Case 1) $p$ is unachievable in the network, which results in $\mathbb{P}\left(\sum_{i=1}^{k} T_{i} \leq T\right) \leq p$ for any relay selection scheme in the $k$ communities. In these cases, according to (15), best-effort forwarding is conducted in each community to ensure the highest achievable delivery ratio.

Case 2) If $p$ is achievable in the network, let $R_{i}^{(\text {opt })}$ be the optimal set of selected relays in the $i$ th community, and $R_{i}$ be the corresponding set of selected relays in our approach. Let $p_{i}^{(\text {opt })}=\mathbb{P}\left(T_{i} \leq \frac{T}{k}\right)$ in the optimal relay selection scheme. If $p_{i}^{\text {(opt) }} \leq p_{i}$, obviously $R_{i}^{(\mathrm{opt})} \subseteq R_{i}$. Otherwise if $p_{i}^{\text {(opt) }}>p_{i}$ and $p_{i} \leq p^{\frac{1}{k}}, R_{i}^{(\mathrm{opt})}=R_{i}$ because best-effort data forwarding is conducted in both cases. Since it is impossible that $p_{i}^{(\mathrm{opt})} \geq p_{i} \geq p^{\frac{1}{k}}$ because in (15) we have $\left(\frac{p}{p_{\text {low }}}\right)^{\frac{1}{k-k} \text { low }} \geq p^{\frac{1}{k}}$, we have $R_{i}^{(\text {opt })} \subseteq R_{i}$ for $\forall i$. Therefore, our relay selection scheme can also achieve the delivery ratio $p$.

Note that, Theorem 4 implicitly assumes that intercommunity destination-awareness is accurately maintained at the data source $S$, and data is successfully carried at all selected relays. However, in practice the gateway paths maintained at $S$ may be out-of-date and suboptimal due to the intermittent network connectivity in DTNs, and data may be dropped by a relay with limited buffer during data item selection described in Section IV-D. As a result, the performance of MDM in practice may deflect from our theoretical expectation, and we will evaluate the performance of our proposed scheme experimentally in Section V.

\section{G. Discussions}

In our approach, social-based metrics analytically represent nodes' capabilities to forward data to its destinations, and our relay selection scheme provides lower performance bounds to ensure that the required multicast performance can be achieved. Nevertheless, these lower bounds may not be tight, and consequently the number of selected relays may not be minimized with respect to the specified multicast performance.

In DTNs, minimizing the multicast cost and achieving the tight performance bounds are generally difficult due to the following two reasons. First, due to the intermittent network connectivity, it is difficult for the data source or gateway nodes to have global network knowledge, and they select relays only based on their limited local knowledge about the destinations. Second, node behaviors in DTNs are highly dynamic, and the knowledge maintained by a node about other nodes may be inaccurate. Therefore, analytically and accurately representing nodes' probabilistic capabilities for forwarding data to its destinations is difficult. In some cases, relaxation is necessary for developing social-based relay selection metrics. For example, in Section III-B, the node contact probability is expressed in (3) in a convolutional form, which requires global network knowledge to calculate, and hence Theorem 1 is used for approximation only based on local network knowledge. Similarly in Section IV-F, we fix $T_{i}=T / k$ to ensure that the cumulative delivery ratio achieves the required $p$. In Section V-D, we will further evaluate the tightness of these performance bounds via trace-driven simulations.

\section{PERFormance EVAluations}

In this section, we compare the performance of our proposed multicast schemes to existing data forwarding schemes including Epidemic [37], PROPHET with the default parameter settings in [26], and binary Spray-and-Wait [34]. We also compare our SDM scheme to social-based unicast schemes including SimBet [10] and BUBBLE Rap [20] to show the essential difference between multicast and unicast in DTNs and compare our SDM scheme to other social-based multicast schemes to show the advantage of our proposed relay selection strategy.

We implemented all schemes at the routing layer, which is independent from the underlying MAC or physical layer protocols. The first $20 \%$ of each trace is the warmup period for nodes to accumulate network information such as contact rates and opportunistic paths. Data is generated and forwarded during the remaining part of the trace.

We use the following metrics for evaluations and only count the delivered destinations that are the destinations having received the data. Each simulation is repeated 500 times with random sources and destinations for statistical convergence.

1) Delivery ratio, the ratio of the number of delivered destinations to the total number of destinations.

2) Actual delay, the average delay for all delivered destinations to receive the data.

3) Average cost, the average number of relays used for one delivered destination to receive a data item.

\section{A. Performance of SDM}

Evaluation of the performance of our SDM scheme uses the Infocom trace, with the required delivery ratio $p=80 \%$. The data is generated at randomized times and multicast to 10 randomly selected destinations. For PROPHET, multicast is separate unicast processes for each destination. For Spray-and-Wait, each simulation run dynamically controls the number $(L)$ of data copies to be the same as the number of relays $(K)$ selected by our SDM scheme.

Due to the low contact frequency in DTNs, relays may be unable to contact the destinations on time, when the time constraint $T$ is short. As a result, the actual delivery ratio shown in Fig. 9(a) highly relates to $T$. When $T$ becomes larger, this ratio increases dramatically, and the average delay increases accordingly from consideration of more "delivered destinations."

The delivery ratio of our centrality-based SDM scheme is also limited by $T$. The required delivery ratio $p$ is unachievable when $T \leq 14 \mathrm{~h}$. In this case, the data source generally selects all of its contacted neighbors as relays, so that multicasts data with best effort. Nevertheless, with different values of $T$, our SDM scheme shows only $10 \%$ degradation in delivery ratio and delay compared to Epidemic, while our scheme outperforms PROPHET and Spray-and-Wait by $25 \%$. Similar results appear 


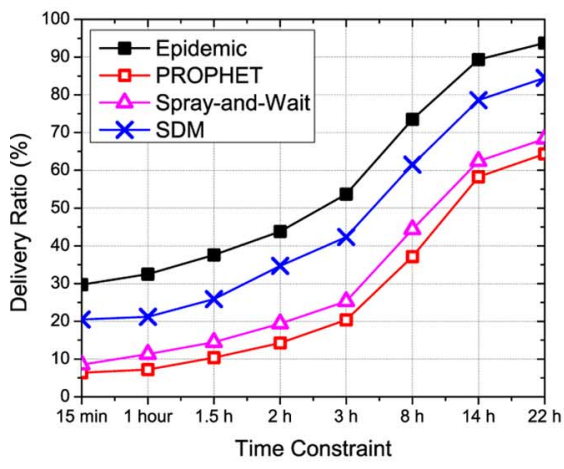

(a)

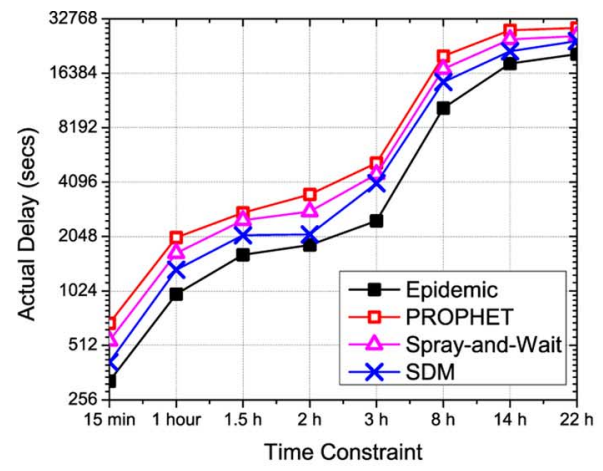

(b)

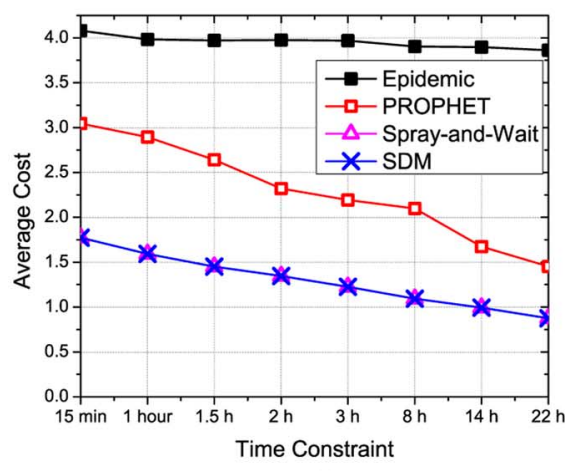

(c)

Fig. 9. Performance of SDM on the Infocom trace. (a) Delivery ratio. (b) Actual delivery delay. (c) Average cost.

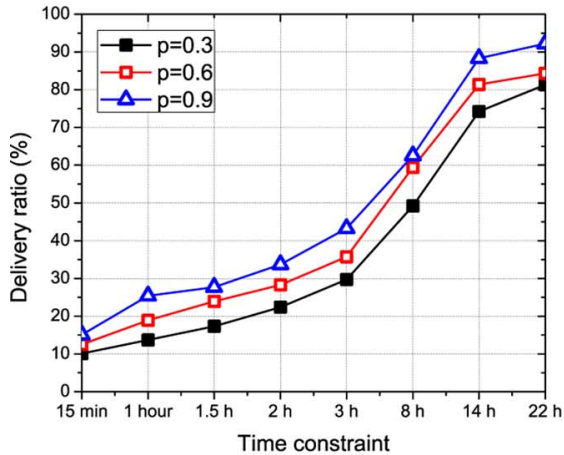

(a)

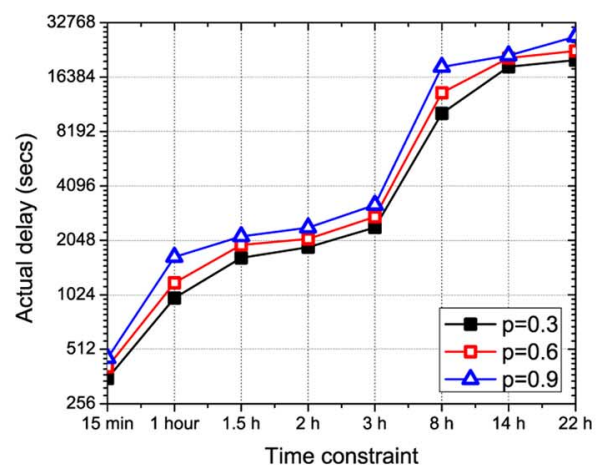

(b)

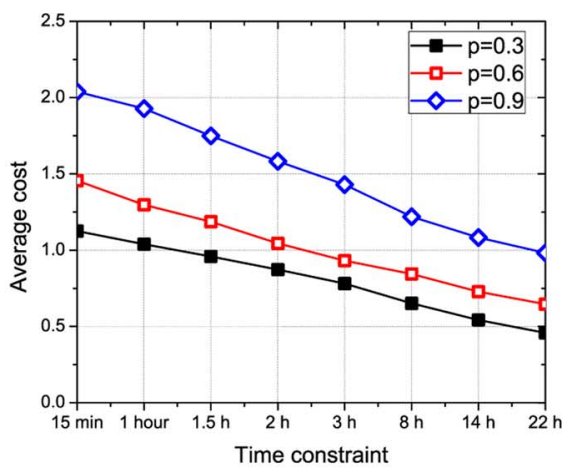

(c)

Fig. 10. Performance of SDM with different required delivery ratio $p$. (a) Delivery ratio. (b) Actual delivery delay. (c) Average cost.

in Fig. 9(b) in terms of delay. The actual delay of SDM is 5\% longer than Epidemic, but is 10\% shorter than PROPHET.

Comparatively, Fig. 9(c) shows that our approach has significantly lower cost than other schemes. When $T$ increases from $15 \mathrm{~min}$ to $22 \mathrm{~h}$, the average cost of SDM is reduced from 1.75 to 1.0 relays. When $T$ is $22 \mathrm{~h}$, the cost of SDM is $66 \%$ of that of PROPHET and $25 \%$ of that of Epidemic. These results show the advantage of our approach to improving cost-effectiveness of multicast relay selection.

Fig. 10 investigates the effects of different requirements of delivery ratio $p$. When $p$ is low, increasing $p$ leads to a considerable improvement for actual delivery ratio, as shown by Fig. 10(a). When $p$ increases from 0.3 to 0.6 , the delivery ratio increases by $15 \%-25 \%$. This improvement becomes smaller when $p$ is high. Higher $p$ also leads to higher cost. Fig. 10(c) shows that when $p$ increases from 0.3 to 0.9 , the average cost increases by $25 \%-30 \%$.

\section{B. Performance of $M D M$}

We use the MIT Reality trace with a larger network scale and dynamic social community structures to evaluate our MDM scheme and detect the social community structures using the $k$-clique method described in Section IV-C. The specified delivery ratio requirement is $p=60 \%$, and the source node generates five data items. The number of destinations for each data item is uniformly randomized in $[3,9]$. The size of each data item is uniformly randomized in $[20 \mathrm{Mb}, 50 \mathrm{Mb}]$, and the buffer size of each node is uniformly randomized in [50 Mb, $100 \mathrm{Mb}$. Each selected relay is able to carry any single data item, but is unable to carry all data items simultaneously. Various conditions of node buffer constraints are simulated by randomizing the node buffer sizes and data sizes.

Since the pairwise contact rates in the MIT Reality trace are much lower (see Table I), the time constraint and actual delay are correspondingly much longer. The delivery ratio shown in Fig. 11(a) highly relates to the time constraint. Our MDM scheme can only achieve the required delivery ratio $p=60 \%$ when the time constraint is longer than 6 weeks, but our MDM scheme keeps similar delivery ratio with Epidemic routing, and outperforms PROPHET by more than $100 \%$ when the time constraint is longer than 1 week. Meanwhile, the average cost of our approach is much lower than that of Epidemic and PROPHET, as shown in Fig. 11(c). For the longest time constraint (6 months), the average cost of our approach is $50 \%$ of that of PROPHET, and $11 \%$ of that of Epidemic.

In Fig. 12, we vary the number of data items $n$ in the network and evaluate the performance of MDM with different node buffer constraints. While Fig. 12(b) and (c) shows trends similar to SDM cases, Fig. 12(a) shows the basic difference between SDM and MDM. In Fig. 12(a), the delivery ratio decreases when $n$ increases. The major reason is that when $n$ increases, there is generally a larger amount of data being forwarded in the network, and the data items compete for the limited buffer of relays. As a result, a data item has higher chance of being dropped from relays, consequently affecting the cumulative delivery ratio.

Moreover, Fig. 13 evaluates the overhead of maintaining network information for MDM, which includes the overhead of maintaining the pairwise contact rates among mobile nodes, information of opportunistic paths, and the social community 


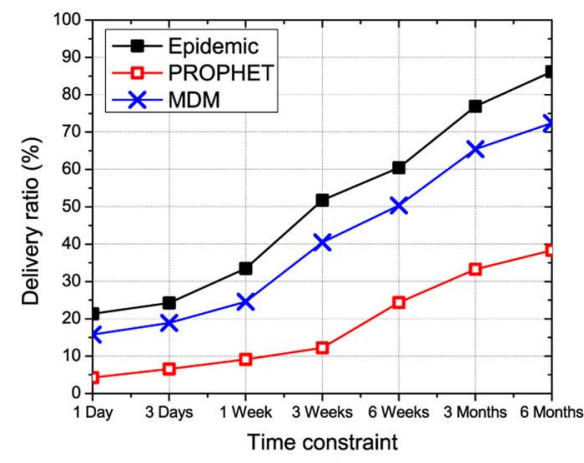

(a)

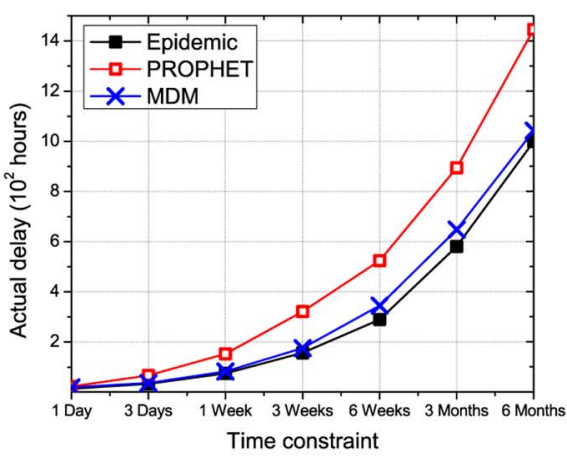

(b)

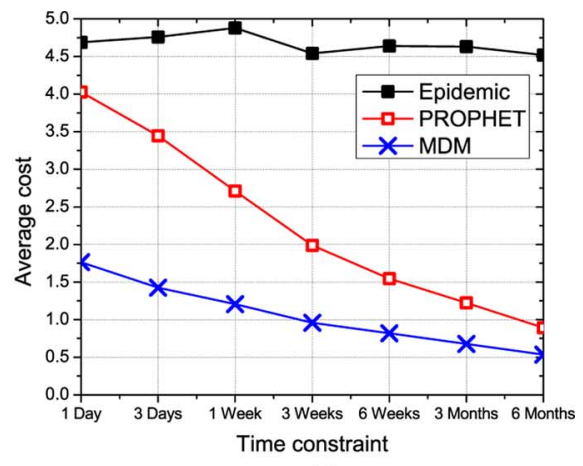

(c)

Fig. 11. Performance of MDM on the MIT Reality trace. (a) Delivery ratio. (b) Actual delivery delay. (c) Average cost.

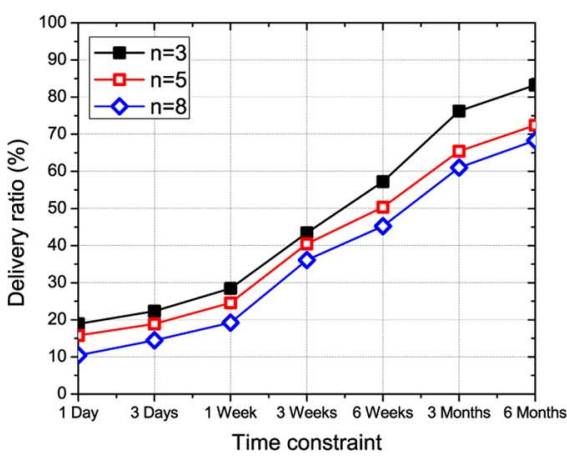

(a)

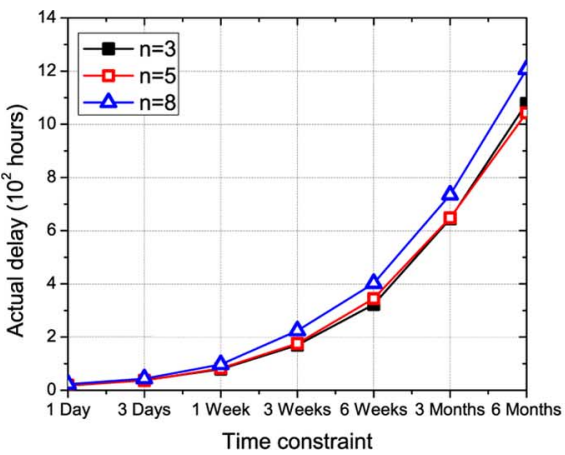

(b)

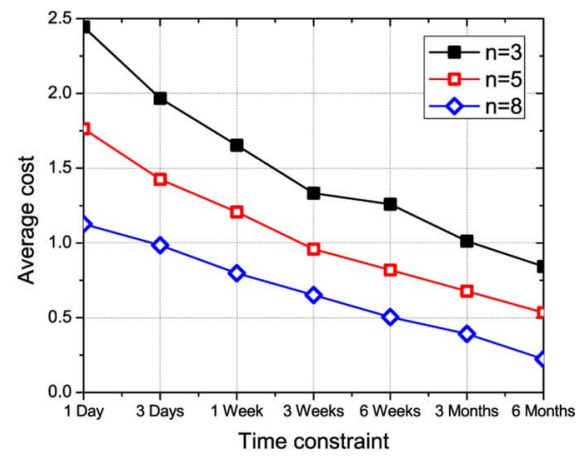

(c)

Fig. 12. Performance of MDM with different numbers of data items $n$. (a) Delivery ratio. (b) Actual delivery delay. (c) Average cost.

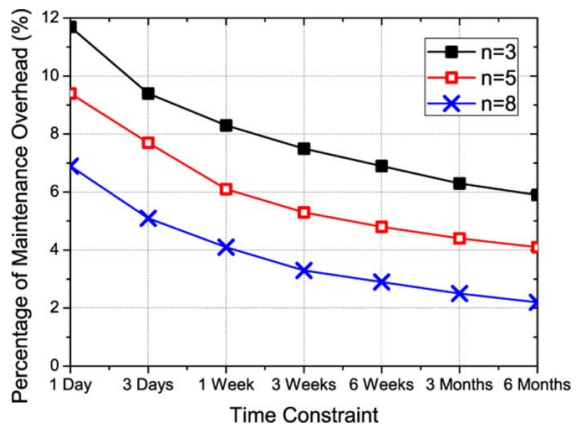

Fig. 13. Overhead of maintaining network information.

structure in the network. This overhead is evaluated as the percentage over the data transmission overhead since the network starts. As shown in Fig. 13, such overhead percentage generally decreases when the time constraint $T$ becomes longer or there are more data items being generated at the data source, resulting in relays forwarding more data copies. We observe that such percentage of overhead in most cases is below $10 \%$, and is even lower than 5\% when $T$ becomes larger. This result shows that our community-based scheme for MDM is able to efficiently reduce the overhead of maintaining destination-awareness, and consequently ensures cost-effectiveness of multicast.

\section{Comparison to Other Social-Based Schemes}

We compare the performance of our SDM scheme to other social-based unicast and multicast schemes using the Infocom trace. In unicast schemes including SimBet [10] and BUBBLE Rap [20], each multicast destination is handled separately. The multicast scheme for comparison is the Social Network Aided Multicast Delivery (SNAMD) [7], where social communities are detected by the $k$-clique method [21]. We use the same simulation setting as in Section V-A.

SimBet calculates the betweenness and similarity metrics and exchanges data between nodes based on their SimBet utilities. SimBet does not consider contact frequencies between node pairs, and therefore leads to lower delivery ratio and longer delay, as shown in Fig. 14(a) and (b). Comparatively, BUBBLE Rap benefits from the social community structure and hierarchical forwarding. It has similar delay to our SDM scheme, but its delivery ratio is $20 \%$ lower than our SDM scheme. This is mainly because in our implementation of BUBBLE Rap, node centrality is approximated by the number of unique nodes being contacted over a time period in the past (S-Window), which, however, does not accurately estimate the nodes' probabilities of contacting others in the future. Comparatively, by selecting relays for multiple destinations simultaneously and considering social community, SNAMD achieves higher delivery ratio with lower forwarding cost. However, its multicast cost-effectiveness is still $20 \%-30 \%$ lower than that of our SDM scheme, as shown in Fig. 14(a) and (c).

\section{Detailed Analysis}

1) Impact of Assumptions: As described earlier, our proposed multicast schemes include specific assumptions of the network contact patterns. We assume that the intercontact time for each pair of nodes is exponentially distributed, and also assume that the pairwise contact rates are stationary over time. In practice, these assumption may not hold in all cases. For example, empirical studies showed that the distribution of pairwise 


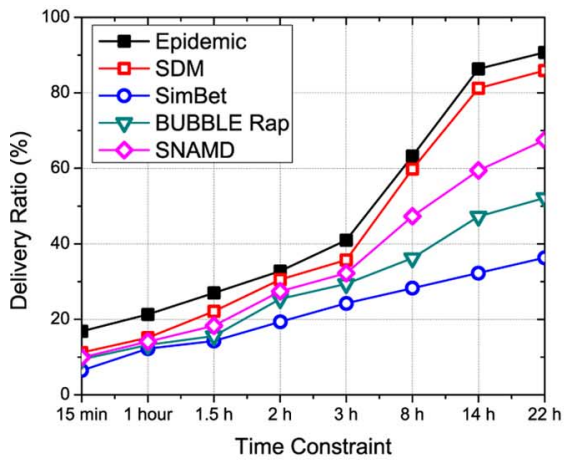

(a)

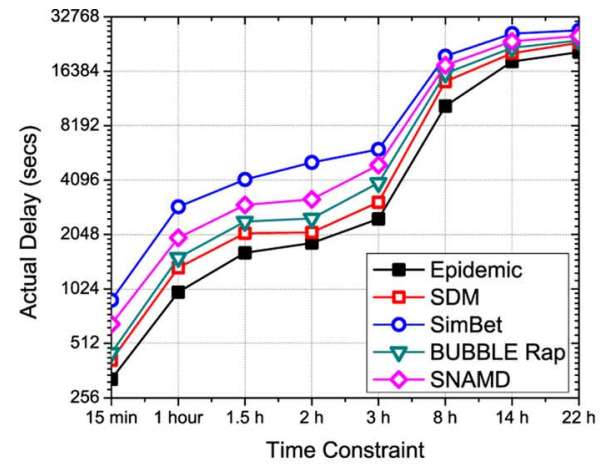

(b)

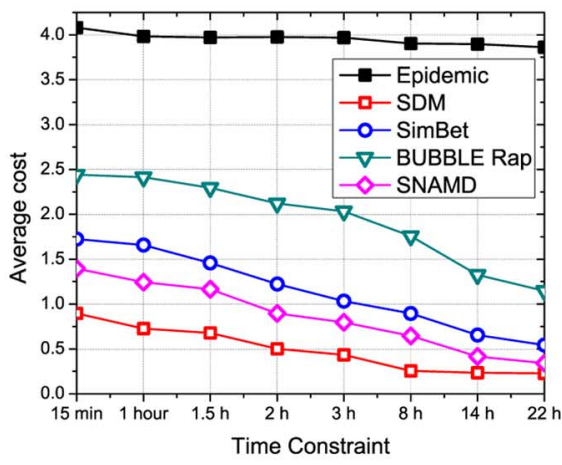

(c)

Fig. 14. Comparison to other social-based schemes. (a) Delivery ratio. (b) Actual delivery delay. (c) Average cost.

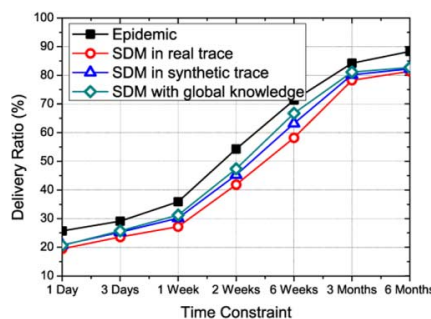

(a)

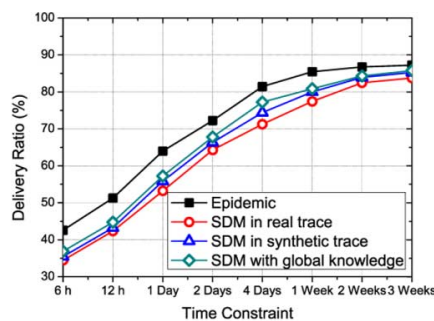

(b)

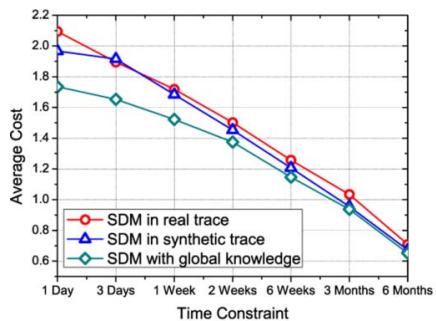

(a)

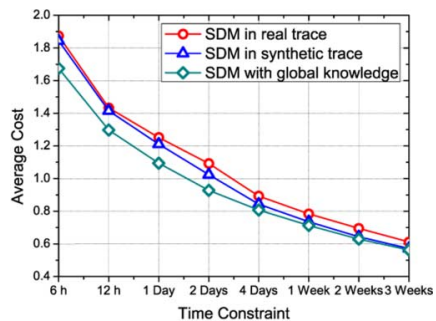

(b)
Fig. 15. Impact of assumptions to the SDM delivery ratio. (a) MIT Reality. (b) UCSD.

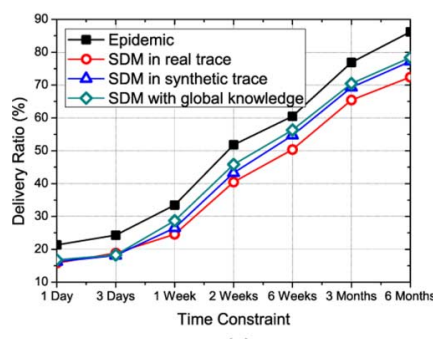

(a)

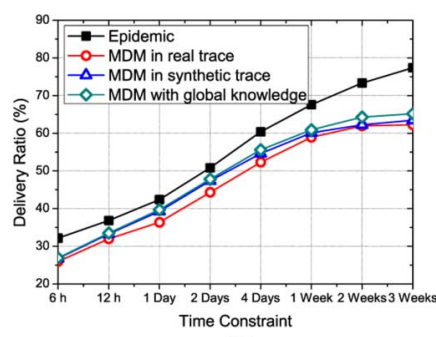

(b)
Fig. 16. Impact of assumptions to the MDM delivery ratio. (a) MIT Reality. (b) UCSD

intercontact time can also be power-law or log-normal in some traces [8].

To evaluate the impact of these assumptions on the multicasting performance, we specifically synthesize node contacts from realistic DTN traces listed in Table I. For each pair of nodes in the network, we cumulatively calculate their pairwise contact rate throughout the entire trace, and then generate synthetic contacts between the two nodes following a Poisson process with the pairwise contact rate as a parameter. We compare the performance of our proposed multicast schemes on both realistic and synthetic traces, and the comparison results are shown in Figs. 15 and 16.

In general, we observe that there are nonnegligible differences on the multicasting performance between realistic and synthetic traces. In the synthetic traces, our proposed metrics for relay selection estimate the contact capabilities of relays with $100 \%$ accuracy, and consequently lead to higher delivery ratio. However, for both SDM and MDM cases in all traces, such increase in delivery ratio is negligible when $T$ is small, and is no larger than $10 \%$ when $T$ is large. Such results essentially show
Fig. 17. Number of relays used for SDM. (a) MIT Reality. (b) UCSD.

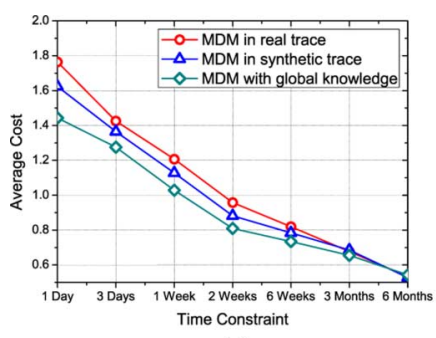

(a)

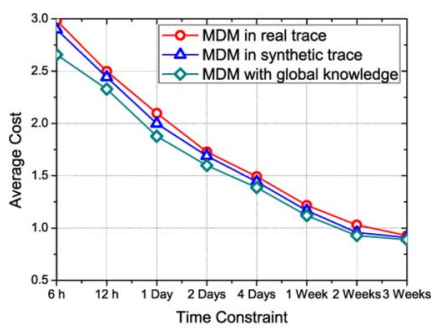

(b)
Fig. 18. Number of relays used for MDM. (a) MIT Reality. (b) UCSD.

that the assumptions we made have only limited impact on multicasting performance in practice.

2) Tightness of Performance Bounds: For both SDM and MDM problems, we proposed relay selection strategies based on the local knowledge of individual nodes and provided theoretical bounds that ensure that the required performance can be achieved by the selected relays. In practice, due to the incompleteness of local network knowledge in DTNs, these performance bounds may not be tight, and hence the relays selected by our schemes may be suboptimal for satisfying the required multicasting performance.

The evaluation of such suboptimality of relay selection, based on the synthetic traces described earlier, assumes that all nodes have global network knowledge. For SDM, the data source calculates the convolution in (3) instead of selecting relays based on Theorem 1. For MDM, the data source maintains the complete opportunistic path to all nodes in the network without applying intercommunity multicast.

The evaluation results are shown in Figs. 17 and 18. When global network knowledge is available, we are able to optimally select the best relays for multicasting, and hence the number of 
selected relays is reduced in both SDM and MDM cases. Nevertheless, such reduction is limited. For SDM cases in Fig. 17, the reduction percentage is lower than $20 \%$ when $T$ is small, and quickly diminishes when $T$ becomes larger. The reduction percentage for MDM cases is even smaller as shown in Fig. 18. These results show that the performance bounds in our schemes provide good approximation to optimality. Therefore, our relay selection strategies are able to significantly improve multicast cost-effectiveness by selecting the appropriate nodes as relays.

\section{RELATED WORK}

In [37], the authors studied Epidemic routing in DTNs, in which the basic idea is to select all nodes in the network as relays. Later studies developed relay selection strategies to approach the performance of Epidemic routing with lower numbers of data copies created in the network. While the most conservative strategy [35] always keeps a single data copy and Spray-and-Wait [34] holds a fixed number of data copies, most relay selection schemes leave such numbers as dynamic and make data forwarding decisions by comparing nodes' metrics for relay selection. For example, in [4] and [10], a relay forwards data to another node whose metric is higher than itself. Delegation forwarding [12] reduces the cost by only forwarding data to the node with the highest metric, and RAPID [1] opportunistically determines data replication based on the marginal data utility. In our scheme, we follow a similar method to determine the number of relays in different ways, with respect to SDM and MDM problems.

The relay selection metrics generally evaluate the capability of a mobile node to forward data to the specified destinations, and various metrics can be applied to the same strategy for different performance requirements. Some schemes predict such capability by estimating the colocation probabilities of mobile nodes based on their mobility patterns in different ways, such as the Kalman filter [9], semi-Markov chains [41], and Hidden Markov models [15]. More specifically, [42] employs some nodes with desirable mobility patterns as message ferries, and [6] analyzes the performance of such mobility-assisted schemes theoretically.

Since the node mobility patterns are highly volatile and difficult to characterize or predict, node contact process [19] is also exploited, as abstraction of node mobility, to calculate relay selection metrics. More specifically, the nodes' capability of contacting others in the future is predicted based on their cumulative contact records from the past. Based on the experimental [6], [23] and theoretical [5] analysis of node contact characteristics, relay selection metrics have been proposed to estimate node contact probability in the future [1], [4], [16], [26]. However, these metrics provide only simple heuristics for selecting relays without performance guarantee.

Node contact process can also be exploited for data delivery from a social network perspective. Most schemes exploit sociological centrality metrics [27] for relay selections. SimBet routing [10] uses an ego-centric betweenness metric, and BUBBLE Rap [20] considers node centrality in a hierarchical manner based on social community knowledge. In both schemes, the network contact graph is binary and therefore cannot differentiate the contact frequency of various pairs of mobile nodes. This network modeling limits the data forwarding performance because node centrality values do not really represent the nodes' capability of contacting others.

As generalization of forwarding data to a single destination, content dissemination in DTNs has also been studied [3], [17], [22]. In [3], efficient utility functions are developed for content dissemination, and [22] investigates optimal rate allocation schemes to maximize the data dissemination speed. Social relations among mobile nodes are also exploited for content dissemination in DTNs. For example, ContentPlace [2] exploits social community structures for data dissemination by defining community-based relay selection policies. Such community knowledge is also used in [40] to develop a social-aware overlay system for effective publish/subscribe communication. SocialCast [9] investigates the homophily phenomenon [29] for publish/subscribe system by assuming that users with common interest contact each other more frequently.

Multicast, on the other hand, can be considered as a special type of data dissemination with respect to a prespecified group of data recipients and has been extensively studied in wireless ad hoc and sensor networks [25], [38]. However, there are limited research efforts on multicast in DTNs due to the difficulty of analytical relay selection for multiple destinations simultaneously. Some initial studies focus on semantic multicast models [43] and multicast capacity analysis [24]. Reference [32] proposes theoretical insights on the local maxima of relay selection, but does not consider the case of multiple data items and the impact of node buffer constraint. Social network concepts have recently been introduced to improve the multicast cost-effectiveness [7], but an analytical model for relay selection remains absent. The models in [18] are not sufficient to analyze nodes' capabilities for forwarding data to multiple destinations, especially for MDM.

\section{CONCLUSION}

In this paper, we studied social-aware multicast in DTNs and exploited social network concepts to improve cost-effectiveness of multicast. We studied multicast with single and multiple data items, developed analytical models for multicast relay selection, and investigated the essential difference between multicast and unicast in DTNs. Trace-driven evaluations show that our approach achieves similar delivery ratio and delay to that of Epidemic routing, but significantly reduces forwarding cost.

\section{REFERENCES}

[1] A. Balasubramanian, B. Levine, and A. Venkataramani, "DTN routing as a resource allocation problem," in Proc. ACM SIGCOMM, 2007, pp. $373-384$

[2] C. Boldrini, M. Conti, and A. Passarella, "Contentplace: Social-aware data dissemination in opportunistic networks," in Proc. MSWiM, 2008, pp. 203-210.

[3] C. Boldrini, M. Conti, and A. Passarella, "Modelling data dissemination in opportunistic networks," in Proc. ACM CHANTS, 2008, pp. 89-96.

[4] J. Burgess, B. Gallagher, D. Jensen, and B. Levine, "MaxProp: Routing for vehicle-based disruption-tolerant networks," in Proc. IEEE INFOCOM, 2006, pp. 1-11.

[5] H. Cai and D. Y. Eun, "Crossing over the bounded domain: From exponential to power-law inter-meeting time in MANET," in Proc. ACM MobiCom, 2007, pp. 159-170.

[6] A. Chaintreau, P. Hui, J. Crowcroft, C. Diot, R. Gass, and J. Scott, "Impact of human mobility on opportunistic forwarding algorithms," IEEE Trans. Mobile Comput., vol. 6, no. 6, pp. 606-620, Jun. 2007.

[7] M. C. Chuah, "Social network aided multicast delivery scheme for human contact-based networks," in Proc. 1st Simplex, 2009, Article no. 3 . 
[8] V. Conan, J. Leguay, and T. Friedman, "Characterizing pairwise intercontact patterns in delay tolerant networks," in Proc. Ist Int. Conf. Autonomic Comput. Commun. Syst., 2007, Article no. 19.

[9] P. Costa, C. Mascolo, M. Musolesi, and G. Picco, "Socially-aware routing for publish-subscribe in delay-tolerant mobile ad hoc networks," IEEE J. Sel. Areas Commun., vol. 26, no. 5, pp. 748-760, Jun. 2008.

[10] E. Daly and M. Haahr, "Social network analysis for routing in disconnected delay-tolerant MANETs," in Proc. ACM MobiHoc, 2007, pp. $32-40$.

[11] N. Eagle and A. Pentland, "Reality mining: Sensing complex social systems," Pers. Ubiquitous Comput., vol. 10, no. 4, pp. 255-268, 2006.

[12] V. Erramilli, A. Chaintreau, M. Crovella, and C. Diot, "Delegation forwarding," in Proc. ACM MobiHoc, 2008, pp. 251-260.

[13] K. Fall, "A delay-tolerant network architecture for challenged internets," in Proc. ACM SIGCOMM, 2003, pp. 27-34.

[14] L. Freeman, "A set of measures of centrality based on betweenness," Sociometry, vol. 40, no. 1, pp. 35-41, 1977.

[15] W. Gao and G. Cao, "Fine-grained mobility characterization: Steady and transient state behaviors," in Proc. ACM MobiHoc, 2010, pp. $61-70$

[16] W. Gao and G. Cao, "On exploiting transient contact patterns for data forwarding in delay tolerant networks," in Proc. IEEE ICNP, 2010, pp. $193-202$.

[17] W. Gao and G. Cao, "User-centric data dissemination in disruption tolerant networks," in Proc. IEEE INFOCOM, 2011, pp. 3119-3127.

[18] W. Gao, Q. Li, B. Zhao, and G. Cao, "Multicasting in delay tolerant networks: A social network perspective," in Proc. ACM MobiHoc, 2009 , pp. 299-308.

[19] W. Hsu and A. Helmy, "On nodal encounter patterns in wireless LAN traces," IEEE Trans. Mobile Comput., vol. 9, no. 11, pp. 1563-1577, Nov. 2010.

[20] P. Hui, J. Crowcroft, and E. Yoneki, "Bubble rap: Social-based forwarding in delay tolerant networks," in Proc. ACM MobiHoc, 2008, pp. $241-250$.

[21] P. Hui, E. Yoneki, S. Chan, and J. Crowcroft, "Distributed community detection in delay tolerant networks," in Proc. ACM/IEEE MobiArch, 2007, Article no. 7.

[22] S. Ioannidis, A. Chaintreau, and L. Massoulie, "Optimal and scalable distribution of content updates over a mobile social network," in Proc. IEEE INFOCOM, 2009, pp. 1422-1430.

[23] T. Karagiannis, J.-Y. Boudec, and M. Vojnovic, "Power law and exponential decay of inter contact times between mobile devices," in Proc. ACM MobiCom, 2007, pp. 183-194.

[24] U. Lee, S.-Y. Oh, K.-W. Lee, and M. Gerla, "RelayCast: Scalable multicast routing in delay tolerant networks," in Proc. IEEE ICNP, 2008, pp. 218-227.

[25] X.-Y. Li, "Multicast capacity of wireless ad hoc networks," IEEE/ACM Trans. Netw., vol. 17, no. 3, pp. 950-961, Jun. 2009.

[26] A. Lindgren, A. Doria, and O. Schelen, "Probabilistic routing in intermittently connected networks," Comput. Commun. Rev., vol. 7, no. 3, pp. 19-20, 2003.

[27] P. Marsden, "Egocentric and sociocentric measures of network centrality," Social Netw., vol. 24, no. 4, pp. 407-422, 2002.

[28] M. McNett and G. Voelker, "Access and mobility of wireless PDA users," Comput. Commun. Rev., vol. 9, no. 2, pp. 40-55, 2005.

[29] M. McPherson, L. Smith-Lovin, and J. Cook, "Birds of a feather: Homophily in social networks," Annu. Rev. Sociol., vol. 27, no. 1, pp. 415-444, 2001.

[30] M. Motani, V. Srinivasan, and P. Nuggehalli, "Peoplenet: Engineering a wireless virtual social network," in Proc. ACM MobiCom, 2005, pp. 243-257.

[31] G. Palla, I. Derényi, I. Farkas, and T. Vicsek, "Uncovering the overlapping community structure of complex networks in nature and society," Nature, vol. 435, no. 7043, pp. 814-818, 2005.

[32] A. Picu and T. Spyropoulos, "Distributed stochastic optimization in opportunistic networks: The case of optimal relay selection," in Proc. ACM CHANTS, 2010, pp. 21-28.

[33] S. M. Ross, Introduction to Probability Models. New York: Academic, 2006

[34] T. Spyropoulos, K. Psounis, and C. Raghavendra, "Spray and wait: An efficient routing scheme for intermittently connected mobile networks," in Proc. ACM SIGCOMM Workshop Delay-Tolerant Netw., 2005, pp. 252-259.

[35] T. Spyropoulos, K. Psounis, and C. Raghavendra, "Efficient routing in intermittently connected mobile networks: The single-copy case," IEEE/ACM Trans. Netw., vol. 16, no. 1, pp. 63-76, Feb. 2008.

[36] V. Srinivasan, M. Motani, and W. Ooi, "Analysis and implications of student contact patterns derived from campus schedules," in Proc. ACM MobiCom, 2006, pp. 86-97.
[37] A. Vahdat and D. Becker, "Epidemic routing for partially connected ad hoc networks,” Duke University, Durham, NC, Tech. Rep. CS-200006 2000 .

[38] Y. Wang, X. Chu, X. Wang, and Y. Cheng, "Optimal multicast capacity and delay tradeoffs in MANETs: A global perspective," in Proc. IEEE INFOCOM, 2011, pp. 640-648.

[39] D. Watts and S. Strogatz, "Collective dynamics of small-world networks," Nature, vol. 393, pp. 440-442, 1998.

[40] E. Yoneki, P. Hui, S. Chan, and J. Crowcroft, "A socio-aware overlay for publish/subscribe communication in delay tolerant networks," in Proc. ACM MSWiM, 2007, pp. 225-234.

[41] Q. Yuan, I. Cardei, and J. Wu, "Predict and relay: An efficient routing in disruption-tolerant networks," in Proc. ACM MobiHoc, 2009, pp. 95-104.

[42] W. Zhao, M. Ammar, and E. Zegura, "A message ferrying approach for data delivery in sparse mobile ad hoc networks," in Proc. ACM MobiHoc, 2004, pp. 187-198

[43] W. Zhao, M. Ammar, and E. Zegura, "Multicasting in delay tolerant networks: Semantic models and routing algorithms," in Proc. SIGCOMM Workshop Delay Tolerant Netw., 2005, pp. 268-275.

[44] H. Zhu, L. Fu, G. Xue, Y. Zhu, M. Li, and L. M. Ni, "Recognizing exponential inter-contact time in VANETs," in Proc. IEEE INFOCOM, 2010, pp. 1-5

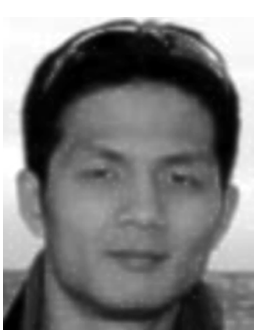

Wei Gao (S'11) received the B.E. degree in electrica engineering from the University of Science and Technology of China, Hefei, China, in 2005, and is currently pursuing the $\mathrm{Ph} . \mathrm{D}$. degree in computer science and engineering at The Pennsylvania State University, University Park.

His research interests include opportunistic mobile network, mobile social network, and user mobility modeling.

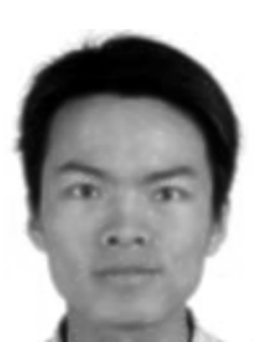

Qinghua Li (S'09) received the B.E. degree from Xi'an Jiaotong University, Xi'an, China, in 2004, and the M.S. degree from Tsinghua University, Beijing, China, in 2007, and is currently pursuing the Ph.D. degree in computer science and engineering at The Pennsylvania State University, University Park.

His research interests include wireless networks and network security.

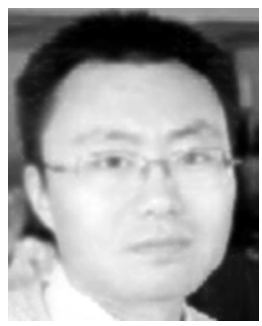

Bo Zhao (S'11) received the B.S. degree in computer science from Peking University, Beijing, China, in 2003, and is currently pursuing the Ph.D. degree in computer science and engineering at The Pennsylvania State University, University Park.

His research interests include $3 \mathrm{G}$ network security, power saving of the telecom networks, and $3 \mathrm{G}$ network services.

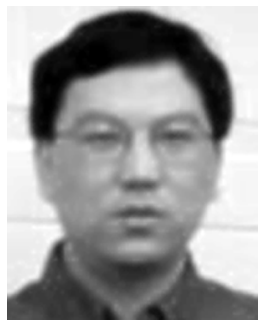

Guohong Cao (S'98-A'99-M'03-SM'07-F'11) received the B.S. degree from Xi'an Jiaotong University, Xi'an, China, in 1990, and the M.S and Ph.D. degrees from The Ohio State University, Columbus, in 1997 and 1999, respectively, all in computer science.

Since then, he has been with the Department of Computer Science and Engineering, The Pennsylvania State University, University Park, where he is currently a Professor. He has published more than 150 papers in the areas of wireless sensor networks wireless network security, vehicular ad hoc networks, data access and dissemination. His research interests are wireless networks and mobile computing.

Prof. Cao has served on the Editorial Board of the IEEE TRANSACTIONS on Mobile Computing, the IEEE Transactions ON Wireless COMMUNICATIONS, and the IEEE TRANSACTIONS ON VEHICULAR TECHNOLOGY. He has served as a program committee member for many conferences. He was a recipient of the NSF CAREER Award in 2001. 